\title{
Shakeup Effects on Photoluminescence from the Wigner Crystal
}

\author{
D.Z. Liu \\ Center for Superconductivity Research, Department of Physics, University of Maryland, College Park, MD 20742 \\ H.A. Fertig \\ Department of Physics and Astronomy, University of Kentucky, Lexington, Kentucky 40506-0055 \\ S. Das Sarma \\ Department of Physics, University of Maryland, College Park, MD 20742
}

(Submitted to Physical Review Letters on 13 June 1994)

\begin{abstract}
We develop a method to compute shakeup effects on photoluminescence in a Wigner crystal from localized holes. Our method treats the lattice electrons and the tunneling electron on an equal footing, and uses a quantummechanical calculation of the collective modes that is realistic throughout the Brillouin zone. We find that shakeup produces a series of sidebands that may be identified with maxima in the collective mode density of states, and definitively distinguishes the crystal state from a liquid state. We also find a shift in the main luminescence peak, that is associated with lattice relaxation in the vicinity of a vacancy.

PACS numbers: 78.20.Ls, 72.20.Jv, 73.20.Dx
\end{abstract}

Sixty years ago, Wigner [1] pointed out that an electron gas will undergo a zero-temperature, quantum phase transition into a crystalline phase as the density is lowered. Forty-five years later, the first convincing evidence of an electron crystal was presented for a system of electrons on a He surface [2]. The electron densities attainable in this fashion are extremely low, however, making this an unattractive system for observing the quantum phase transition. Semiconductors are much more attractive systems in this sense, because one has great control over the electron densities, through dopant concentrations. A particularly good candidate for observing the Wigner crystal (WC) is the two-dimensional electron gas (2DEG), as realized in modulation doped semiconductors. Samples of this type are now available with such high quality that the electron groundstate is not necessarily dominated by disorder. The possibility of observing the $\mathrm{WC}$ is further enhanced by the application of a strong perpendicular magnetic field, which quenches the kinetic energy, and allows the formation of a crystal state at higher densities (for which disorder effects are less important) than would be possible without it.

Experimental evidence for the WC in 2DEG's has accumulated over the last several years [3]. One probe that has produced much intriguing data is photoluminescence $(\mathrm{PL})$, in which either a valence band hole [4] or a hole bound to an acceptor [5.6] recombines with an electron in the 2DEG, producing a characteristic photon spectrum. A mean-field analysis [7] of the latter type of experiment showed that the PL spectrum has, in principle, characteristic signatures of the WC: a "Hofstadter butterfly" [8] spectrum for the case of weak interactions between the electrons and the hole, and a characteristic shift in the PL spectrum upon melting of the crystal.

In this Letter, we go beyond the mean-field approximation, to examine shakeup effects on the PL spectrum; i.e., we will examine how the collective mode spectrum of the WC (which, at long wavelengths, corresponds to the classical phonon spectrum), and the fact that some of these modes may be excited in the electron-hole recombination process, modify the results of the mean-field theory. We will consider in detail only the case of a localized hole [5,6]. Our method is purely quantum-mechanical, and treats both the tunneling electron and the other lattice electrons on the same footing. Furthermore, we employ a quantum treatment of the collective modes to realistically account for contributions both from small and large wavevector collective excitations of the lattice. Since we are working in the strong magnetic field limit, we consider only excitations within the lowest Landau level (LLL). Our main results are: (1) Shakeup effects shift the main PL peak to higher energies than found in a mean-field treatment [7]. (2) The Hofstadter spectrum is eliminated from the PL spectrum, even in the case of weak electronhole interactions [6] (although we will argue below that it survives in the itinerant hole case (7). The sudden shift of the PL spectrum upon melting, by contrast, survives even when shakeup is included. (3) Phonon sidebands appear that correspond to maxima in the phonon density of states (DOS); some (but not all) of these sidebands are results of van Hove singularities in the DOS, and so are characteristic of an ordered WC state. For the case of weak electron-hole interactions [6], we do not see these sidebands in the liquid state, so that phonon satellites uniquely distinguish between a liquid and a solid state. Interestingly, for stronger electron-hole interactions, a shakeup satellite persists even above the melting temperature. We expect this sideband to lose oscillator strength relative to the main peak, either with increasing temperature or decreasing electron-hole interaction strength. The latter may be accomplished by examining PL from several samples with different acceptor - 2DEG 
setback distances [6].

Examples of our calculated PL spectra are shown in Fig. 1 for filling fractions $\nu=1 / 5$ and $\nu=2 / 7$, for electron density $N_{s}=6 \times 10^{10} \mathrm{~cm}^{-2}$. Our hole is assumed to be strongly localized, and located $250 \AA$ from the electron plane. For the case of no electron-hole interaction [Fig. 1(a)], at low temperature, a well-defined shakeup peak may be seen approximately $2 \mathrm{meV}$ below the main PL peak; a second very weak satellite is observed approximately $3.5 \mathrm{meV}$ below the main peak. The origins of these peaks may be understood in terms of the phonon DOS, which is illustrated in Fig. 2. A van Hove singularity, arising from zone-edge phonons, appears as a strong double peak near $0.4 \mathrm{meV}$. Two other peaks may be seen near $1.2 \mathrm{meV}$ and $1.9 \mathrm{meV}$. There are weak sidebands associated with each of these peaks in the PL spectrum. The precise interpretation of these peaks is unclear; however, it has been speculated that these represent vacancyinterstitial excitations 10. We point out that it is crucial to use a fully quantum mechanical treatment of the collective excitations of the lattice to observe these higher order satellites; classical treatments of the phonons [9.11 do not produce these unusual excitations.
FIG. 1. PL spectra for $(\mathrm{a}) \nu=1 / 5, T=0$, no electron-hole interaction; (b) $\nu=1 / 5, T=0$, with electron-hole interaction; (c) $\nu=2 / 7, T=0$, no electron-hole interaction. Inset: PL spectra for (a) and (b), with $T$ just above the melting temperature. In order to distinguish different spectra, they were seperated by 250 units.

It should also be noted that the splitting between the main PL peak and the first sideband is actually larger than the energy at which the van Hove singularity in the phonon DOS appears. The reason for this is that there is a strong self-energy renormalization due to the phonons in the main PL peak. Physically, this arises because the final state of the crystal contains a vacancy, which is lowered in energy by a distortion of the lattice - i.e., by allowing the electrons surrounding the vacancy to relax inward. The self-energy shift accounts for this lowering in energy of the final state of the $\mathrm{WC}$, and leads to an upward shift of the main PL peak. Our calculation shows that the final states in which phonons are excited are not nearly so strongly renormalized by lattice relaxation effects, leading to the increased splitting between the main PL peak and the sidebands.

The inset to Fig. 1 illustrates the PL spectrum in the melted state both without and with an electronhole interaction. In the former case, there is no phonon sideband present. This is necessarily so, because in the melted phase, the density is uniform, and there are no collective modes in the LLL [10]. By contrast, when an electron-hole interaction is present, there is a nonuniform electron density near the hole, allowing some local collective modes to persist even above the melting temperature. With further increase in temperature, or increased setback between the hole and the 2DEG, the oscillator strength of this mode will significantly decrease.

Fig. 1(c) illustrates our results for $\nu=2 / 7$ in the absence of electron-hole interactions. As can be seen, except for a change in energy scale caused by changing the magnetic field, the lineshape is essentially identical to the case of $\nu=1 / 5$. This contrasts sharply with the results found in the mean-field approximation [7], where

FIG. 2. Collective mode DOS for $\nu=1 / 5, T=0$.

without electron-hole interactions, a filling $\nu=p / q$ generally yields $p$ distinct lines for a localized hole. While the splittings are so small in that situation that they are difficult in practice to resolve, evidently shakeup effects wipe out this structure even in principle. We note that for the case of an itinerant hole, we expect these characteristic splittings to survive shakeup effects. The reason is that (neglecting excitonic effects), the PL spectrum is related to the product of Green's functions for the hole and the electron. For localized holes, only the latter has poles in the form of a Hofstadter spectrum at the meanfield level, which are wiped out by shakeup effects. However, for itinerant holes, the hole Green's function also has poles of the Hofstadter form, which are unaffected by shakeup. Thus, one should in principle be able to 
identify this characteristic spectrum of bands and gaps that is unique to a WC in a magnetic field in itinerant hole experiments 12.

We now present an outline of how these results were derived; a more detailed description will be presented in a future publication [13]. Previously [7], it was shown that if we consider a superlattice of holes, with $n_{e}$ electrons and one hole per unit cell, the PL intensity may quite generally be written in the form $P(\omega) \propto \operatorname{Im}[R(\omega+i \delta)]$, where $R(\omega)=\left(n_{h} \Omega / 2 \pi l_{0}^{2}\right) \sum_{\mathbf{G}} R(\mathbf{G}, \omega) e^{-G^{2} l_{0}^{2} / 4}$, and we have approximated the core-hole wavefunctions as delta-functions, $n_{h}$ is the density of holes, $\Omega$ the volume of the system, and the vectors $\mathbf{G}$ are the reciprocal lattice vectors of the superlattice. The magnetic length $l_{0}=(\hbar c / e B)^{1 / 2}$ will be set to unity in the remainder of this paper. $R(\mathbf{G}, \omega)$ is defined as $R(\mathbf{G}, \omega)=(1 / g) \sum_{X} e^{-i G_{x} X+i G_{x} G_{y} / 2} R_{i i}\left(X, X-G_{y} ; \omega\right)$, where $g$ is the Landau level degeneracy, $X$ is the guiding center quantum number, and

$$
R_{i j}\left(X_{1}, X_{2} ; i \omega_{n}\right)=-\int_{0}^{\beta}<T_{\tau} a_{X_{1}}(\tau) c_{i}(\tau) c_{j}^{\dagger} a_{X_{2}}^{\dagger}>e^{i \omega_{n} \tau} d \tau
$$

with $a_{X}^{\dagger}$ creating an electron in state $X$ and $c_{i}^{\dagger}$ creating a hole in the unit cell $i$, and $\beta$ is the inverse temperature. Working in the lowest Landau level, the equation of motion for $R_{i j}(\mathbf{G}, \omega)$ may be written as

$$
\begin{aligned}
\frac{\partial}{\partial \tau} R_{i j}(\mathbf{G}, \tau) & =<\rho(\mathbf{G}, \tau)>\delta_{i j} \delta(\tau)-\epsilon_{0} R_{i j}(\mathbf{G}, \tau)-n_{h} \sum_{\mathbf{G}^{\prime}} V\left(\mathbf{G}^{\prime}\right) e^{i \mathbf{G}^{\prime} \times \mathbf{G} / 2-G^{\prime 2} / 4} R_{i j}\left(\mathbf{G}-\mathbf{G}^{\prime}, \tau\right) \\
& -\frac{1}{\Omega} \sum_{\mathbf{q} \neq 0} v(\mathbf{q}) C_{i j}(-\mathbf{q}, \mathbf{q}+\mathbf{G}) e^{i \mathbf{G} \times \mathbf{q} / 2-q^{2} / 2}-\frac{1}{\Omega} \sum_{\mathbf{q}} V(\mathbf{q}) C_{i j}(-\mathbf{q}, \mathbf{G}) e^{-i \mathbf{q} \cdot \mathbf{R}_{i}-q^{2} / 4}
\end{aligned}
$$

In Eq.(1),$v(\mathbf{q})$ and $V(\mathbf{q})$ are the Fourier transforms of the electron-electron and electron-hole interactions, respectively, $\epsilon_{0}$ is the energy of the localized hole, and the sum over $\mathbf{G}^{\prime}$ is only over reciprocal lattice vectors, while the sums over $\mathbf{q}$ are over all wavevectors. $\mathbf{R}_{i}$ specifies the position of the hole in the $i$ th unit cell, and $\left\langle\rho(\mathbf{G}, \tau)>e^{-G^{2} / 4}\right.$ is the expectation value of a Fourier component of the electron density. While this quantity is independent of $\tau$ in the groundstate, it will be convenient for later purposes to formally leave it as an argument of the density. The method for computing these Fourier components has been described elsewhere [7] 10 . The correlation function $C_{i j}\left(\mathbf{p}_{1}, \mathbf{p}_{2}\right)$ in the last two terms is just Fourier transformation of the following correlation function:

$$
C_{i j}\left(X_{1} X_{2} ; X_{3} X_{4} ; \tau^{\prime}, \tau\right) \equiv-g<T_{\tau} a_{X_{1}}^{\dagger}\left(\tau^{\prime}\right) a_{X_{2}}\left(\tau^{\prime}\right) a_{X_{3}}(\tau) c_{i}(\tau) c_{j}^{\dagger}(0) a_{X_{4}}^{\dagger}(0)>.
$$

Eq.(11) represents the first in an infinite series of equations relating an $n$ particle Green's function to the $n+1$ particle Green's function [14]. In the mean-field approximation, it is simplified by employing a Hartree-Fock (HF) decomposition of Eq.(2), which converts Eq.(1) into a self-consistent equation for $R_{i j}$ [7]. To include shakeup effects, we instead extend this hierarchy to one more level, writing down a self-consistent form for $C_{i j}$ which explicitly contains the collective mode excitations. To carry out this program, it is convenient to implicitly define a self-energy by substituting the last two terms in Eq. (11) by $-\sum_{\mathbf{G}^{\prime}} \int_{0}^{\beta} d \tau^{\prime} \Sigma\left(\mathbf{G}, \mathbf{G}^{\prime} ; \tau-\tau^{\prime}\right) R_{i j}\left(\mathbf{G}^{\prime}, \tau^{\prime}\right)$. The HF approximation for the PL is equivalent to taking $\Sigma\left(\mathbf{G}, \mathbf{G}^{\prime} ; \tau-\tau^{\prime}\right)$ by $\Sigma^{H F}\left(\mathbf{G}, \mathbf{G}^{\prime}\right) \delta\left(\tau-\tau^{\prime}\right)$, where

$$
\Sigma^{H F}\left(\mathbf{G}, \mathbf{G}^{\prime}\right)=W\left(\mathbf{G}-\mathbf{G}^{\prime}\right)<\rho\left(\mathbf{G}-\mathbf{G}^{\prime} ; \tau\right)>e^{i \mathbf{G} \times \mathbf{G}^{\prime} / 2}+\frac{1}{2 \pi} \sum_{\mathbf{q}} V(\mathbf{q})<\rho(-\mathbf{q}, \tau)>e^{-q^{2} / 4-i \mathbf{q} \cdot \mathbf{R}_{i}} \delta_{\mathbf{G}, \mathbf{G}^{\prime}}
$$

where $W$ is the sum of the direct and exchange Coulomb potentials [7, 10]. To generate a self-consistent equation for $C_{i j}$, we take a functional derivative [14] of Eq.(11) with respect to a spatially periodic external potential $U\left(\mathbf{p}, \tau^{\prime}\right)$. In doing this, it must be noted that a term directly coupling the Green's function $R_{i j}$ to the external potential $U$ must be added to Eq.(11), and the sums over reciprocal lattice vectors must be extended to all wavevectors, because $U$ is not in general commensurate with the lattice. After an arduous calculation, the result expresses $C_{i j}$ in terms of the density-density correlation function $\chi\left(\mathbf{G}_{1}+\mathbf{q}, \mathbf{G}_{2}+\mathbf{q} ; \tau\right) \equiv \chi_{\mathbf{G}_{1} \mathbf{G}_{2}}(\mathbf{q} ; \tau)=-g<T \tilde{\rho}\left(\mathbf{G}_{1}+\mathbf{q}, \tau\right) \tilde{\rho}\left(-\mathbf{G}_{2}-\mathbf{q} ; 0\right)>$. The Fourier transform of this response function contains poles at the collective mode frequencies of the system; it is thus through $\chi$ that shakeup effects are introduced. We have computed this function numerically using a generalized random phase approximation [10] which does not assume small displacements of the lattice electrons (as is necessary in classical approaches [9]), and thus gives a realistic dispersion relation for the collective modes across the entire Brillouin zone. Upon substituting the resulting $C_{i j}$ into Eq.(1) , one obtains the self-energy $\Sigma=\Sigma^{H F} \delta\left(\tau-\tau^{\prime}\right)+\delta \Sigma$, with 


$$
\begin{aligned}
\delta \Sigma\left(\mathbf{G}_{1}, \mathbf{G}_{2}, \tau_{1}-\tau_{2}\right)= & \frac{1}{4 \pi} \sum_{\mathbf{G}_{1}^{\prime} \mathbf{G}_{2}^{\prime}} \int_{B Z} d^{2} \mathbf{q} e^{i \mathbf{q} \times\left(\mathbf{G}_{1}-\mathbf{G}_{2}\right) / 2-i \mathbf{G}_{1} \times \mathbf{G}_{1}^{\prime} / 2+i \mathbf{G}_{2} \times \mathbf{G}_{2}^{\prime} / 2} W\left(\mathbf{G}_{1}^{\prime}+\mathbf{q}\right) W\left(\mathbf{G}_{2}^{\prime}+\mathbf{q}\right) \\
& \times F\left(\mathbf{G}_{1}-\mathbf{G}_{1}^{\prime}-\mathbf{q}, \mathbf{G}_{2}-\mathbf{G}_{2}^{\prime}-\mathbf{q} ; \tau_{1}-\tau_{2}\right) \chi_{\mathbf{G}_{1}^{\prime} \mathbf{G}_{2}^{\prime}}\left(\mathbf{q} ; \tau_{1}-\tau_{2}\right) \\
+ & \frac{1}{(2 \pi)^{3}} \sum_{\mathbf{G}_{1}^{\prime} \mathbf{G}_{2}^{\prime}} \int_{B Z} d^{2} \mathbf{q} e^{-\left(\mathbf{G}_{1}^{\prime}+\mathbf{q}\right)^{2} / 4-\left(\mathbf{G}_{2}^{\prime}+\mathbf{q}\right)^{2} / 4} V\left(\mathbf{G}_{1}^{\prime}+\mathbf{q}\right) V\left(\mathbf{G}_{2}^{\prime}+\mathbf{q}\right) \\
& \times F\left(\mathbf{G}_{1}, \mathbf{G}_{2} ; \tau_{1}-\tau_{2}\right) \chi_{\mathbf{G}_{1}^{\prime} \mathbf{G}_{2}^{\prime}}\left(\mathbf{q} ; \tau_{1}-\tau_{2}\right)
\end{aligned}
$$

In Eq.(4), $\int_{B Z} d^{2} \mathbf{q}$ represents an integral over wavevectors in the first Brillouin zone of the superlattice, and $F$ is a generalized Green's function satisfying the equation of motion

$$
\begin{aligned}
\frac{\partial}{\partial \tau} F\left(\mathbf{G}, \mathbf{G}_{1}, \tau-\tau_{1}\right) & =\delta_{\mathbf{G G}_{1}} \delta\left(\tau-\tau_{1}\right)-n_{h} \sum_{\mathbf{G}^{\prime}} V\left(\mathbf{G}^{\prime}\right) e^{i \mathbf{G}^{\prime} \times \mathbf{G} / 2-G^{\prime 2} / 4} F\left(\mathbf{G}-\mathbf{G}^{\prime}, \mathbf{G}_{1}, \tau-\tau_{1}\right) \\
& -\epsilon_{0} F\left(\mathbf{G}, \mathbf{G}_{1}, \tau-\tau_{1}\right)-\sum_{\mathbf{G}^{\prime}} \Sigma^{H F}\left(\mathbf{G}, \mathbf{G}^{\prime}\right) F\left(\mathbf{G}^{\prime}, \mathbf{G}_{1}, \tau-\tau_{1}\right),
\end{aligned}
$$

Eq.(5) may be solved using methods discussed previously [7:10]. With this expression, we are now able to compute the PL intensity. We substitute $\Sigma=\Sigma^{H F} \delta\left(\tau-\tau^{\prime}\right)+\delta \Sigma$ into Eq.(何), and Fourier transform this with respect to imaginary time. This means that a Fourier transform of Eq.(14) will be necessary, leading to frequency summations of the form (suppressing wavevector arguments) $\sum_{i \omega_{n}} F\left(i \omega_{n}\right) \chi\left(\mathbf{q} ; i \omega-i \omega_{n}\right)$. To accomplish this, we represent $\chi$ as a sum over its collective mode poles [10]; the frequency sums may be then computed using standard methods [14]. The computation of $\delta \Sigma$ is clearly the bottleneck in this computation, since it requires two reciprocal lattice sums and an approximate sum over the continuous wavevector $\mathbf{q}$. We have accomplished this using $469 \mathbf{q}$ points in the first Brillouin zone, for one and three electrons per unit cell, which give very similar results. Finally, once we have computed $\delta \Sigma$, it is straightforward to substitute this into the frequency version of Eq.(何), obtain $R(\mathbf{G}, \omega)$, and from there compute the PL spectrum.

In summary, we have developed a method by which shakeup effects in the PL spectrum of a WC from localized holes may be computed, that treats the tunneling electron and the lattice electrons on an equal footing, and uses a fully quantum treatment of the collective modes that is realistic over the entire Brillouin zone. Our method is quite general, and should be applicable to other shakeup problems where quantum fluctuations are important. We find that the Hofstadter spectrum found in a mean-field analysis of this experiment is lost (although we expect it to survive in itinerant hole experiments), and is replaced by a series of sidebands due to creation of phonons and other collective excitations of the WC. These sidebands are a unique signature of the WC, and can in principle be used to distinguish between a liquid and crystal state of the electrons. We find that there is a sudden shift in the PL spectrum upon melting of the crystal.

Acknowledgments. The authors thank Dr. René Côté for helpful discussions. This work was supported by the NSF, through Grant Nos. DMR 92-02255 and DMR 91-23577, and by the US-ONR. HAF acknowledges the support of the Alfred P. Sloan Foundation and the Research Corporation, through a Cottrell Scholar Award.

[1] E.P. Wigner, Phys. Rev. 46, 1002 (1934)

[2] C.C. Grimes and G. Adams, Phys. Rev. Lett. 42, 795 (1979)

[3] For a review of recent experiments, see B. McCombe and A. Nurmikko, eds., Electronic Properties of Two-Dimensional Systems, (North-Holland, New York, 1994)

[4] S.A. Brown et al., Surface Science 305, 42 (1994); E.M. Goldys et al., Phys. Rev. B 46, 7957 (1992); R.G. Clark, Physica Scripta T39, 45 (1991) and references therein.

[5] H. Buhmann et al., Phys. Rev. Lett. 66, 926 (1991); I.V. Kukushkin et al, Phys. Rev. B 45, 4532 (1992)

[6] I.V. Kukushkin et al., Phys. Rev. Lett. 72, 3594 (1994)

[7] H.A. Fertig, D.Z. Liu, and S. Das Sarma, Phys. Rev. Lett. 70, 1545 (1993); D.Z. Liu, H.A. Fertig, and S. Das Sarma, Phys. Rev. B 48, 11184 (1993); H.A. Fertig, D.Z. Liu, and S. Das Sarma, Surface Science 305, 67 (1994)

[8] D. Hofstadter, Phys. Rev. B 14, 2239 (1976)

[9] P. Johansson and J.M. Kinaret, Phys. Rev. Lett. 71, 1435 (1993)

[10] R. Côté and A.H. MacDonald, Phys. Rev. Lett. 65, 2662 (1990); Phys. Rev. B 44, 8759 (1991)

[11] L. Bonsall and A.A. Maradudin, Phys. Rev. B 15, 1959 (1977)

[12] This conclusion may be modified if the hole is close enough to the electron layer that excitonic effects are important.

[13] H.A. Fertig, D.Z. Liu, and S. Das Sarma, unpublished.

[14] L.P. Kadanoff and G. Baym, Quantum Statistical Mechanics, (Benjamin, Reading, 1981) 